Hypoglycemia-Sensing Neurons of the Ventromedial Hypothalamus Require AMPK-Induced Txn2 Expression but Are Dispensable for Physiological Counterregulation

\author{
Simon Quenneville, ${ }^{1}$ Gwenaël Labouèbe, ${ }^{1}$ Davide Basco, ${ }^{1}$ Salima Metref, ${ }^{1}$ Benoit Viollet, ${ }^{2}$ Marc Foretz, ${ }^{2}$ and \\ Bernard Thorens ${ }^{1}$
}

Diabetes 2020;69:2253-2266 | https://doi.org/10.2337/db20-0577

\begin{abstract}
The ventromedial nucleus of the hypothalamus (VMN) is involved in the counterregulatory response to hypoglycemia. VMN neurons activated by hypoglycemia (glucoseinhibited [GI] neurons) have been assumed to play a critical although untested role in this response. Here, we show that expression of a dominant negative form of AMPK or inactivation of AMPK $\alpha 1$ and $\alpha 2$ subunit genes in Sf1 neurons of the VMN selectively suppressed GI neuron activity. We found that Txn2, encoding a mitochondrial redox enzyme, was strongly downregulated in the absence of AMPK activity and that reexpression of Txn2 in Sf1 neurons restored GI neuron activity. In cell lines, Txn2 was required to limit glucopenia-induced reactive oxygen species production. In physiological studies, absence of GI neuron activity after AMPK suppression in the VMN had no impact on the counterregulatory hormone response to hypoglycemia or on feeding. Thus, AMPK is required for GI neuron activity by controlling the expression of the antioxidant enzyme Txn2. However, the glucose-sensing capacity of VMN GI neurons is not required for the normal counterregulatory response to hypoglycemia. Instead, it may represent a fail-safe system in case of impaired hypoglycemia sensing by peripherally located glucose detection systems that are connected to the VMN.
\end{abstract}

The brain requires a continuous supply of glucose as a source of metabolic energy. This imposes that blood glucose concentrations never fall below the euglycemic level of $\sim 5 \mathrm{mmol} / \mathrm{L}$. In healthy individuals, hypoglycemia does not usually occur because multiple counterregulatory mechanisms rapidly induce the secretion of hormonesglucagon, epinephrine, glucocorticoids, growth hormonewhich together induce hepatic glucose production, suppress insulin secretion, and reduce insulin action on peripheral tissues to restore normoglycemia and glucose availability to the brain (1). However, in insulin-treated patients with type 1 or type 2 diabetes, iatrogenic hypoglycemia is frequently observed, and antecedent hypoglycemia increases the risk to develop subsequent hypoglycemic episodes of higher severity, due to progressive impairments in counterregulatory hormone secretion (2).

The counterregulatory hormone response to hypoglycemia is triggered in large part by glucose-sensing cells of the nervous system (3). These cells regulate the activity of the autonomous nervous system, which is involved in the control of insulin, glucagon, and epinephrine secretion and of glucose production by the liver. They also activate the hypothalamo-pituitary-adrenal axis, which controls glucocorticoid secretion. Glucose-responsive neurons are present in many brain regions, including in the hypothalamus, the brainstem, and the thalamus (3-5). These neurons fall into two categories, glucose-excited (GE) neurons, whose firing rate increases in response to a rise in extracellular glucose concentration, and glucose-inhibited (GI) neurons, which are activated by hypoglycemia (6) and are thought to trigger the counterregulatory response to hypoglycemia.

The ventromedial nucleus (VMN) of the hypothalamus plays an important role in this counterregulatory response, as evidenced by the observation that secretion of glucagon in response to insulin-induced hypoglycemia can be blocked by intra-VMN injection of glucose and that

\footnotetext{
${ }^{1}$ Center for Integrative Genomics, University of Lausanne, Lausanne, Switzerland 2Université de Paris, Institut Cochin, CNRS, INSERM, Paris, France

Corresponding author: Bernard Thorens, bernard.thorens@unil.ch

Received 28 May 2020 and accepted 18 August 2020
}

This article contains supplementary material online at https://doi.org/10.2337/ figshare.12827294.
S.Q. and G.L. equally contributed to this work.

(C) 2020 by the American Diabetes Association. Readers may use this article as long as the work is properly cited, the use is educational and not for profit, and the work is not altered. More information is available at https://www.diabetesjournals .org/content/license. 
2-deoxy-D-glucose (2DG) injection in the VMN of normoglycemic animals is sufficient to stimulate glucagon secretion $(7,8)$. More recently, it has been shown that inactivation of the vesicular glutamate transporter ( $v$ Glut2) gene in Sf1 neurons, which represent most of the VMN neurons, suppresses glutamatergic synaptic transmission and prevents the normal counterregulatory response to hypoglycemia (9). Also, the optogenetic activation of Sf1 neurons induces, whereas their silencing suppresses glucagon secretion (10). There is, thus, strong support for a role of VMN neurons in the control of glucagon secretion.

However, VMN neurons are part of a multisynaptic circuit that includes an afferent and an efferent limb (11). The afferent limb comprises glucose-sensing neurons located outside of the blood-brain barrier, such as those present in the hepatoportal vein area (3) or in the nucleus of the tractus solitarius and which respond to small variations in blood glucose concentration $(5,12)$, and neurons located within the blood-brain barrier, such as the GI neurons of the lateral parabrachial nucleus, which form direct synaptic contacts with VMN neurons (13). The efferent limb, which regulates pancreatic $\alpha$-cell secretion, involves projections from the VMN to the bed nucleus of the stria terminalis, the periaqueductal gray, and preautonomic regions of the brainstem (14). In this circuit, it is usually assumed that hypoglycemia sensing by VMN GI neurons plays an essential role in triggering the counterregulatory response. However, supporting evidence is only circumstantial, based on various, non-cell-specific pharmacological or gene-silencing approaches (15-17). Thus, the relative importance in triggering the counterregulation response of VMN GI neurons and of GI neurons present at other locations of the afferent limb is not established (11).

Activation of VMN GI neurons by hypoglycemia has been proposed to require the presence of AMPK, which recruits a nitric oxide synthase-soluble guanylate cyclase pathway to amplify AMPK activity; this leads to the closure of the CFTR chloride channel and neuron firing (18). Here, we aimed at identifying the role of the AMPK $\alpha 1$ and $\alpha 2$ catalytic subunits in the glucose responsiveness of VMN Sf1 neurons using combination of genetic, electrophysiological, and physiological studies. We showed that GI neurons are no longer detected when AMPK is inactivated. We identified Txn2, encoding a mitochondrial redox protein, as one of the most downregulated genes when AMPK is suppressed and showed that reexpression of Txn2 in Sf1 neurons is sufficient to restore the presence of GI neurons. Finally, we showed that mice with selective inactivation of VMN GI neurons activity had normal counterregulatory response to hypoglycemia and feeding behavior, suggesting that the glucose sensitivity of these neurons is dispensable for the counterregulatory response.

\section{RESEARCH DESIGN AND METHODS}

\section{Mice}

AMPK $\alpha 1^{\text {lox/lox }}, A M P K \alpha 2^{\text {lox/lox }}$ mice (19) were crossed with Sf1-cre mice (9) to generate $A M P K \alpha 1^{\text {lox/lox }} \alpha 2^{\text {lox/lox }}$ and
Sf1-cre;AMPK $\alpha 1^{\text {lox/lox }} \alpha 2^{\text {lox/lox }}$ mice. Mice were on a C57BL/6 background. All studies used littermates as controls. Mice were age-matched and randomly assigned to experimental groups. Animals were housed on a 12-h light/dark cycle and fed with a standard chow (Diet 3436; Provimi Kliba AG). All procedures were approved by the Veterinary Office of Canton de Vaud (Switzerland).

\section{Mouse Genotyping}

Mouse genotyping was performed by PCR analysis (Supplementary Fig. 1). Primers for AMPK $\alpha 1^{\text {lox/lox }}$ were $\mathrm{P} 1 \alpha 1$ (5'-ATT AAA CAC CAC TAA TTG GAA AAC ATT CCC-3') and P2 $\alpha 1$ (5'-GGG CAA GTA AGG CCT GCA GCC CTA CAC TGA-3'), and AMPK $\alpha 1 \Delta$ : P $\alpha 1$ and P3 $\alpha 1$ (5'-GAC CTG ACA GAA TAG GAT ATG CCC AAC CTC-3'). AMPK $\alpha 2^{\text {lox/lox }}$ PCR primers were P1 $\alpha 2$ (5'-GTT ATC AGC CCA ACT AAT TAC AC- $3^{\prime}$ ) and P2 $\alpha 2$ (5'-GCT TAG CAC GTT ACC CTG GAT GG-3'), and AMPK $\alpha 2 \Delta$ : P3 $\alpha 2$ (5'-TTG GCG CTG TCT AGA TCA GGC TTG C-3') and P $4 \alpha 2$ (5'-GTG CTT CCT AAC TGC AGA TGC AGT G-3').

Analysis of genetic recombination in brain regions was performed with DNA extracted (DNeasy Kit, cat: 69504; Qiagen) from tissue punches (punch, ref. 18036; Fine Science Tools) prepared from 1-mm-thick brain sections.

\section{Viral Vectors}

All recombinant AAV constructs were produced at the Gene Therapy Center at the University of North Carolina (Chapel Hill, NC). Viruses were pAAV8-EF1a-Flex-DN-AMPK-K45RT2A-mCherry ( $\alpha 2$ subunit mutant, AAV8-DIO-DN-AMPK) (20), pAAV8-EF1a-Flex-CA-AMPK-H150R-T2A-mCherry (AAV8-DIO-CA-AMPK) (20), pAAV8-FLEX-EGFPL10a (AAV8DIO-L10-EGFP) (21), rAAV8-EF1a-DIO-Txn2-P2A-EGFP (AAV8-DIO-Txn2-EGFP), and rAAV8-EF1a-DIO-P2A-EGFP (AAV8-DIO-EGFP). The last two virus constructs were produced in the laboratory. Lentivectors lenti-hPGK-DNAMPK-K45R-T2A-mCherry and lenti-hPGK-CA-AMPK-H150RT2A-mCherry; lenti-U6-shTxn2-DIO-EGFP were produced as described (22).

\section{Stereotactic Injection of Viruses}

This procedure was performed as described (4) using 6- to 12-week-old mice. Bilateral stereotactic injections in the VMN used the following coordinates: AP 1.3/ML $\pm 0.6 /$ DV $-5.3 \mathrm{~mm}$. A total of $200 \mathrm{~nL}$ of the virus preparations $\left(10^{11}-10^{14}\right.$ viral genomes $\left./ \mathrm{mL}\right)$ were injected in each hemisphere at a rate of $0.1 \mu \mathrm{L} / \mathrm{min}$.

\section{Electrophysiology}

Mice (8-12 weeks old) were deeply anesthetized with isoflurane before decapitation, and $250-\mu \mathrm{m}$ coronal sections containing VMN were prepared using a vibratome (VT1000S; Leica). Electrophysiological recordings were conducted as previously described (5). Whole-cell recordings were performed in current-clamp mode using a MultiClamp 700B amplifier associated with a 1440A Digidata digitizer (Molecular Devices). Neurons with an access 
resistance $>25 \mathrm{M} \Omega$ or changed by $>20 \%$ during the recording were excluded. A hyperpolarization step $(-20 \mathrm{pA}$, $500 \mathrm{~ms}$ ) was applied every $30 \mathrm{~s}$ to measure membrane resistance. Membrane potential and neuronal firings were monitored over time at different extracellular glucose concentrations after a 10-15 min baseline. Signals were digitized at $10 \mathrm{kHz}$, collected, and analyzed using the pClamp 10 data acquisition system (Molecular Devices).

\section{Translating Ribosome Affinity Purification}

Translating ribosomes affinity purification (TRAP) was performed as described (23). Sf1-cre mice were injected in the VMN with AAV8-DIO-L10-EGFP (21). The VMN were microdissected, and pools of six VMNs were constituted. Anti-green fluorescent protein (GFP) antibody (cat 11814460001; Sigma-Aldrich) was used to immunoprecipitate RNAs from Sf1-positive cells. RNAs were amplified by single primer isothermal amplification with the Ovation RNA-Amplification System V2 (NuGEN), providing DNA libraries for RNA sequencing (RNA-seq). After reads were aligned, read counts were summarized with htseq-count (v. 0.6.1) (24) using Mus musculus GRCm38.82 gene annotation. Library sizes were scaled using TMM (trimmed mean of $\mathrm{M}$ ) normalization (EdgeR package version 3.16.3). To evaluate enrichment and depletion of control genes, a moderated $t$ test was used comparing all six inputs compared with all six outputs.

\section{GT1-7 Cells}

GT1-7 cells were cultured in DMEM containing $25 \mathrm{mmol} / \mathrm{L}$ glucose, $10 \%$ FBS, and 5\% horse serum. Cells were transduced with lentivectors at a multiplicity of infection of 500 , yielding $\sim 95 \%$ transduction efficiency. Then, $5 \times 10^{5}$ cells/well were plated in six-well dishes. For superoxide production measurements, GT1-7 cells were incubated in $0.1 \mathrm{mmol} / \mathrm{L}$ or $30 \mathrm{mmol} / \mathrm{L}$ glucose for $48 \mathrm{~h}$ before adding MitoSOX (cat: M36008; Thermo Fisher). After $45 \mathrm{~min}$, cells were trypsinized and analyzed using a FACS (BD Accuri C6; BD Bioscience). Western blot and real-time quantitative PCR (qPCR) analysis were performed as previously described (25). Rabbit antibodies against ACC (cat 3662; Cell Signaling), anti-phospho-Ser79-ACC (cat 3661; Cell Signaling), anti-Txn2 (cat ab185544; Abcam), and anti-actin (cat A2066; Sigma Aldrich) were used. The secondary antibody was a goat horseradish peroxidasecoupled anti-rabbit antibody (cat: NA934; GE Healthcare). Real-time qPCR analysis was performed using the following primers for Txn2 ( $5^{\prime}$-TTC CCT CAC CTC TAA GAC CCT-3', 5'-CCT GGA CGT TAA AGG TCG TCA-3') and actin (5'-CTA AGG CCA ACC GTG AAA AGA T-3', 5'-CAC AGC CTG GAT GGC TAC GT-3').

\section{Glucose and Insulin Tolerance Tests, Glucagon Measurements, and Hypoglycemic Clamps}

Glucose tolerance tests $(2 \mathrm{~g} / \mathrm{kg}$, i.p.) were performed in 15 -h fasted mice and insulin tolerance tests $(0.8$ units $/ \mathrm{kg}$, i.p.) with 6-h fasted mice, as previously described (26).
Plasma glucagon concentrations were measured by ELISA (Mercodia, Uppsala, Sweden) from blood collected by submandibular puncture under isoflurane anesthesia into tubes containing aprotinin/EDTA. Plasma catecholamines were determined by liquid chromatography-tandem mass spectrometry (27). Hypoglycemic clamps were performed as previously described (28) in 5-h fasted mice.

\section{Food Intake}

Continuous food consumption was measured in a 12chamber Oxymax system (Columbus Instruments, Columbus, $\mathrm{OH}$ ). Measurements of feeding initiation after a fast were performed with mice food-deprived for $15 \mathrm{~h}$ and placed in individual cages with weighted food pellets, which were weighted at the indicated intervals.

\section{Statistics}

Values are reported as mean \pm SEM. Data were analyzed with GraphPad Prism software (GraphPad Software, San Diego, CA). Statistical significance was assessed using appropriate statistical tests that are mentioned in each figure's legend.

\section{Data and Resource Availability}

RNA-seq data have been deposited in the Gene Expression Omnibus database under accession number GSE153872. The data sets and reagents generated during and/or analyzed during the current study are available from the corresponding author upon reasonable request.

\section{RESULTS}

\section{Glucose-Sensing by Sf1 VMN Neurons}

To identify Sf1 neurons in brain slices, we injected an AAVDIO-EGFP in the VMN of Sf1-cre mice (Fig. 1A). The electrical activity of cells expressing EGFP was then characterized by whole-cell patch-clamp recordings in the presence of $2.5 \mathrm{mmol} / \mathrm{L}$ and $0.5 \mathrm{mmol} / \mathrm{L}$ glucose. These concentrations represent those found in the brain parenchyma in normoglycemic and hypoglycemic states, respectively (29); these have been routinely used to identify GE and GI neurons (30). Of the analyzed Sf1 neurons, $43 \%$ were $G E$ (Fig. $1 B$ and $C$ ) characterized by their decreased firing activity during hypoglycemia associated with a hyperpolarization $(-54.9 \pm 1.0 \mathrm{mV}$ vs. $-65.7 \pm 1.1 \mathrm{mV}$ in $2.5 \mathrm{mmol} / \mathrm{L}$ and $0.5 \mathrm{mmol} / \mathrm{L}$ glucose, respectively; $P<$ $0.001)$ (Fig. $1 D$ ) and lower membrane resistance (957.7 \pm $81.8 \mathrm{M} \Omega$ vs. $535.9 \pm 54.3 \mathrm{M} \Omega$ in $2.5 \mathrm{mmol} / \mathrm{L}$ and $0.5 \mathrm{mmol} / \mathrm{L}$ glucose, respectively; $P<0.001$ ) (Fig. $1 E$ ). GI neurons comprised 23.3\% of the recorded neurons (Fig. $1 B)$. At $0.5 \mathrm{mmol} / \mathrm{L}$ glucose, firing activity was increased, membrane potential was reduced $(-68.9 \pm 1.3 \mathrm{mV}$ vs. $-58.7 \pm 1.7$ in $2.5 \mathrm{mmol} / \mathrm{L}$ and $0.5 \mathrm{mmol} / \mathrm{L}$ glucose, respectively; $P<0.001$ ), and membrane resistance was increased $(601.7 \pm 74.8 \mathrm{M} \Omega$ vs. $846.4 \pm 94.3 \mathrm{M} \Omega$ in $2.5 \mathrm{mmol} / \mathrm{L}$ and $0.5 \mathrm{mmol} / \mathrm{L}$ glucose, respectively; $P<$ 0.01 ) (Fig. 1H). Of note, the membrane potential and membrane resistance of GE and GI neurons in normoglycemic 


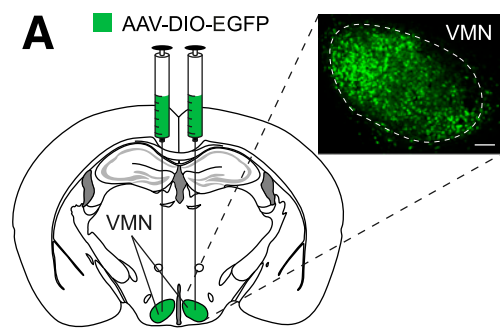

Sf1-cre mouse

C

\section{GE neurons}

Glc $2.5 \mathrm{mmol} / \mathrm{L} \quad 0.5 \mathrm{mmol} / \mathrm{L} \quad 2.5 \mathrm{mmo} / \mathrm{L}$

$$
\downarrow
$$

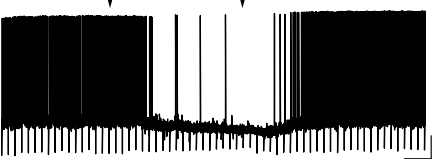

$\mathbf{F}$

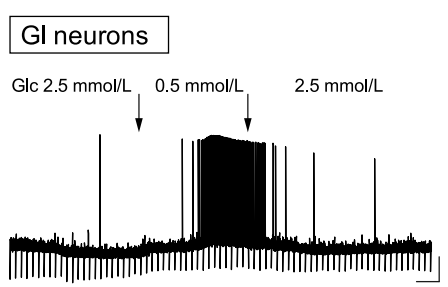

I

\section{NR neurons}

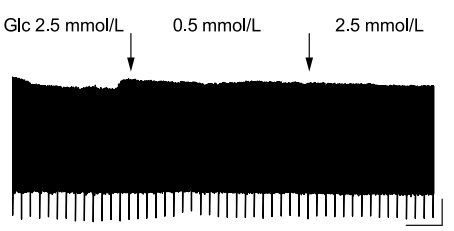

B

GE neurons

GI neurons

NR neurons

D

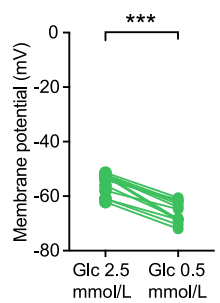

G

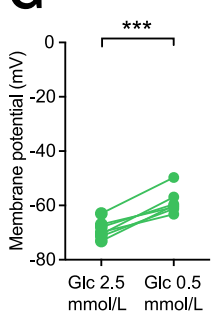

J

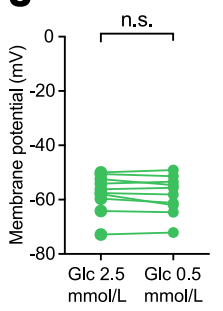

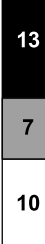

$43.3 \%$

$23.3 \%$

$33.3 \%$

E

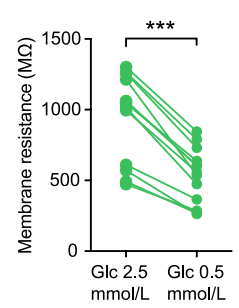

\section{$\mathbf{H}$}

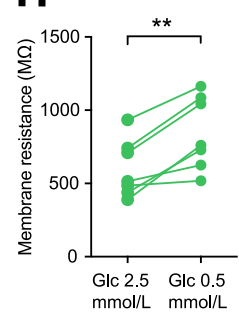

K

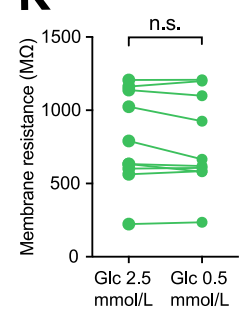

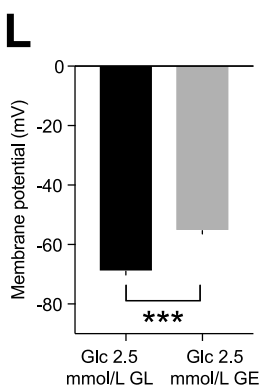

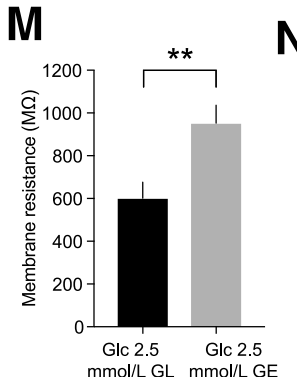

$\mathbf{N}$

GE neurons

GI neurons
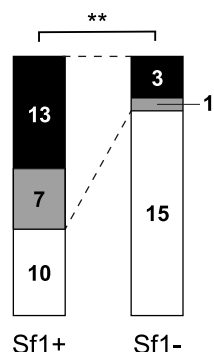

Figure 1-VMN Sf1 neurons are glucose responsive. A: rAAV8-DIO-EGFP was bilaterally injected in the VMN of Sf1-cre mice. Inset shows EGFP expression in the VMN. Scale bar, $100 \mu \mathrm{m}$. B: Distribution of GE ( $n=13$ neurons in nine mice), GI $(n=7$ neurons in five mice), and NR $(n=10$ neurons in nine mice) Sf1 neurons in the VMN. C: GE neurons show decreased activity upon lowering the extracellular glucose to $0.5 \mathrm{mmol} / \mathrm{L}$. This is accompanied by a significant reduction in membrane potential $(D)$ and membrane resistance $(E)$. Gl neurons display increased activity during hypoglycemia $(F)$, with a significant rise in their membrane potential $(G)$ and membrane resistance $(H)$. Other neurons were defined as nonresponder neurons (NR; panel $I$ ) because neither their activity nor their membrane potential $(J)$ or membrane resistance $(K)$ varied upon extracellular glucose variations. Before-after graphs show individual values. Two-tailed paired $t$ test was used. ${ }^{\star} P<0.05$, ${ }^{\star *} P<0.01$, and ${ }^{* \star *} P<0.001$. Under normoglycemia, Gl and GE neurons exhibit significantly different membrane potentials and membrane resistances. GI neurons have a more negative membrane potential than GE neurons $(L)$ and a lower membrane resistance $(M)$. Two-tailed $t$ test was used. ${ }^{\star} P<0.05,{ }^{\star \star} P<0.01$, and ${ }^{\star \star \star} P<0.001$. N: Sf1-negative neurons of the VMN contain fewer glucose-sensing neurons than Sf1 neurons. The Fisher exact test was used. $P=0.0031$ for $\mathrm{Gl}+\mathrm{GE}$ proportion comparison. 
conditions were significantly different, suggesting different complements of ion channels involved in glucosesensing (Fig. $1 L$ and $M)(P<0.01)$. Finally, $33.3 \%$ of the recorded neurons did not change their membrane potential and/or membrane resistance upon changes in glucose concentrations and were classified as nonresponding (NR) neurons (Fig. 1I-K). We also characterized the glucose responsiveness of non-Sf1 neurons of the VMN. Only $21 \%$ were glucose responsive, with a predominance of GE neurons (Fig. $1 N$ ). Thus, the Sf1 neurons of the VMN, which form most of the VMN neurons, comprise twothirds of glucose responsive neurons, and $\sim 40 \%$ of those are GI neurons.

\section{AMPK $\alpha 1$ and $\alpha 2$ Subunits Are Required for GI Neuron Response}

In a first approach to test the role of AMPK in the glucose responsiveness of the VMN neurons, we expressed a dominant negative form of AMPK in Sf1 neurons. This was achieved by stereotactic injection of an AAV-DIO-DNAMPK-mCherry in the VMN of Sf1-cre mice (Fig. $2 A$ and $B$ ). Electrophysiological recordings of the transduced neurons, identified by their red fluorescence, revealed the presence of GE and NR neurons, but no GI neurons could be detected (Fig. $2 C-I$ ).

In a second approach, we generated Sf1-cre;AMPK $\alpha 1^{\text {lox/lox }}$ $\alpha 2^{\text {lox/lox }}$ mice, which displayed efficient recombination of the $A M P K \alpha 1$ and $A M P K \alpha 2$ genes only in the VMN (Supplementary Fig. 1). To identify Sf1 neurons, an AAVDIO-EGFP was injected in the VMN (Fig. 2J), and electrophysiological recordings of these neurons confirmed that inactivation of $A M P K \alpha 1$ and $A M P K \alpha 2$ suppressed the GI neurons (Fig. 2K). GE and NR neurons were still present in the same proportions as found using the DN-AMPK approach. In addition, the reexpression of $A M P K \alpha 1$ and $A M P K \alpha 2$ genes in VMN of those mice allowed the reappearance of GI neurons (Supplementary Fig. 2).

We next tested which of the $\alpha 1$ and $\alpha 2$ AMPK subunits was required for the GI response. We generated Sf1cre;AMPK $\alpha 1^{\text {lox/lox }}$ and Sf1-cre;AMPK $\alpha 2^{\text {lox/lox }}$ mice, injected an AAV-DIO-EGFP in their VMN, and performed electrophysiological analysis of EGFP-labeled Sf1 neurons (Fig. $3 A-D)$. Inactivation of the $A M P K \alpha 1$ or $A M P K \alpha 2$ gene did not suppress the presence of GI neurons, and the distribution of GE, GI, and NR neurons was not significantly different from that of control mice (Fig. $3 A-D)(P>0.05)$.

Finally, we investigated whether overexpression of a constitutively active form of AMPK in Sf1 neurons, using an AAV-DIO-AMPK-CA-mCherry, would modify the proportion of GE and GI neurons. However, when overexpressed in Sf1 neurons, it did not affect the proportion of GE, GI, and NR neurons (Fig. $3 E$ and $F)(P>$ 0.05).

Taken together, the above data showed that the response of Sf1 GI neurons to hypoglycemia depends on AMPK but that the $\alpha 1$ and the $\alpha 2$ subunits have redundant roles. Furthermore, the expression of a constitutively active form of AMPK in Sf1 neurons does not change the distribution of GE, GI, and NR neurons, indicating that activation of AMPK is not sufficient to convert NR or GE neurons into GI neurons. This further implies that GI neurons have specific glucose-signaling mechanisms in which AMPK activity must play a specific role.

\section{Thioredoxin 2 Is an AMPK-Regulated Gene Required for the GI Response}

To search for potential regulators of the GI neuron response to hypoglycemia, we used a TRAP approach to selectively characterize the transcriptome of Sf1 neurons. Sf1-cre mice were injected in the VMN with an AAV-DIOmCherry or an AAV-DIO-AMPK-DN-mCherry, and both groups of mice received at the same time an AAV-DIOL10-EGFP encoding a L10 ribosomal protein-GFP fusion protein that integrates in Sf 1 neurons ribosomes. The day before the experiment, the mice were fasted overnight. Their VMNs were then dissected out, lysed, and the ribosomes immunoprecipitated with an anti-GFP antibody. RNA-seq was then performed on the immunoprecipitated ribosomal fraction (output) and on the nonimmunoprecipitated (input) material. RNA-seq data from control and AMPK-DN overexpressing VMN were first combined and analyzed to confirm that the immunoprecipitated fractions were enriched in mRNAs known to be expressed in the VMN (Nr5a1 [Sf1], Fezf1, Sox14, Gpr149) (31) and depleted in mRNAs expressed by non-VMN neurons (Agrp, Npy, Scl16a11 [Gat-3], Th), glial cells (ApoE, Gfap, Opalin), and oligodendrocytes (Olig1, Plp1, Mal) (Fig. 4A). Then, for each mRNA, we measured their enrichment in the immunoprecipitated fraction (ratio of output vs. input) and calculated how these ratios differ in Sf1 neurons expressing or not the AMPK-DN. These data are presented in the volcano plot of Fig. $4 B$.

Among the dysregulated genes, we focused on the downregulation of mitochondrial thioredoxin 2 (Txn2). This downregulation was observed in the three RNA samples from neurons expressing the AMPK-DN (Fig. $4 C$ ) and was confirmed by real-time qPCR analysis of mRNAs immunoprecipitated from a second TRAP experiment (Fig. 4D). Txn2 is a mitochondrial enzyme involved in redox reactions $(32,33)$, which can modulate mitochondrial respiration (34) and apoptosis (35). It also participates in the regulation of reactive oxygen and nitrogen species that are increased after insulin-induced hypoglycemia and which may control the response of glucosesensitive neurons $(16,18,36,37)$.

To assess whether Txn2 participates in the response of GI neurons to hypoglycemia, we transduced Sf1 neurons of $S f 1$-cre;AMPK $\alpha 1^{\text {lox/lox }} \alpha 2^{\text {lox/lox }}$ mice with an AAV-DIOTxn2-EGFP virus (Fig. $5 A$ and $B$ ). Electrophysiological analysis of the transduced neurons revealed that Txn2 overexpression restored the presence of GI neurons (Fig. $5 G-I)$, which displayed membrane depolarization (59.9 \pm $2.7 \mathrm{mV}$ vs. $65.6 \pm 2.3 \mathrm{mV}$ in $2.5 \mathrm{mmol} / \mathrm{L}$ and $0.5 \mathrm{mmol} / \mathrm{L}$ glucose, respectively; $P<0.01$ ) and increased membrane 


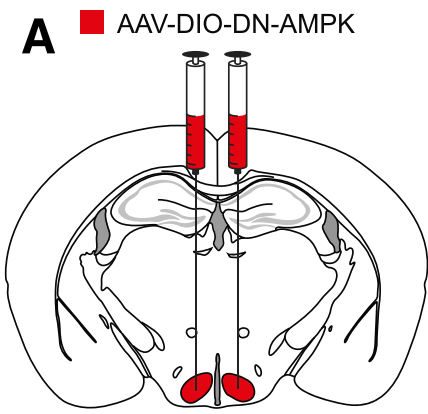

Sf1-cre mouse

D

\section{GE neurons}

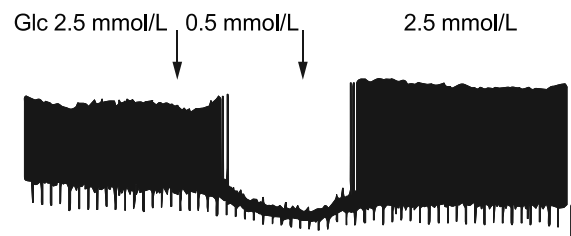

G
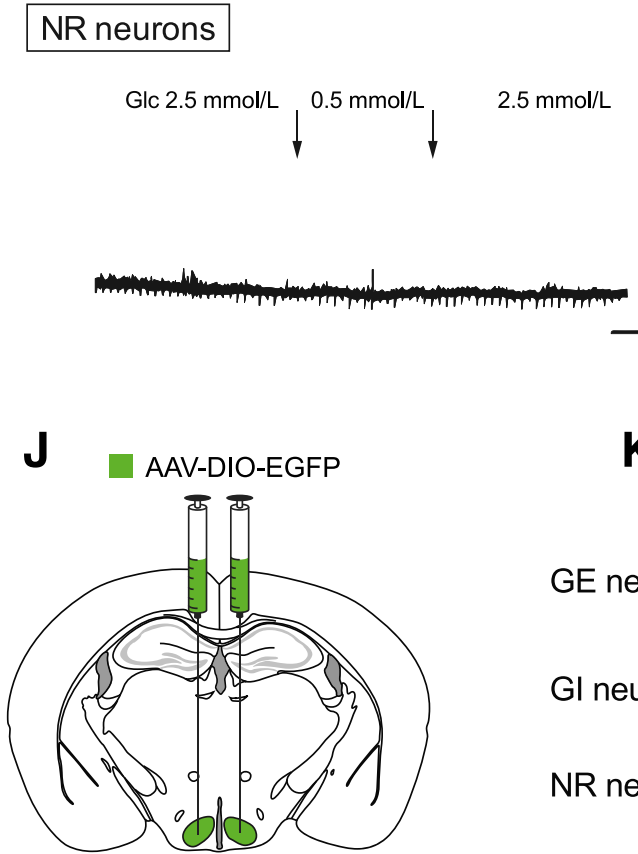

Sf1-cre;AMPK $\alpha 1^{10 x / l o x} \alpha 2^{\text {lox/lox }}$ mouse

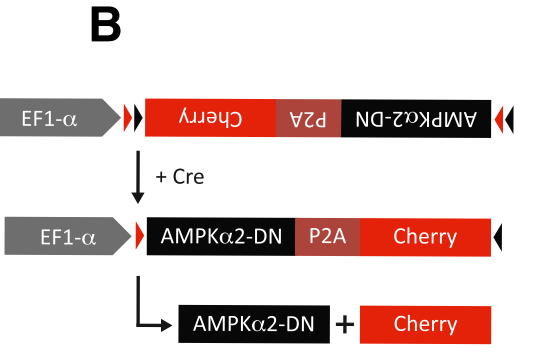

E
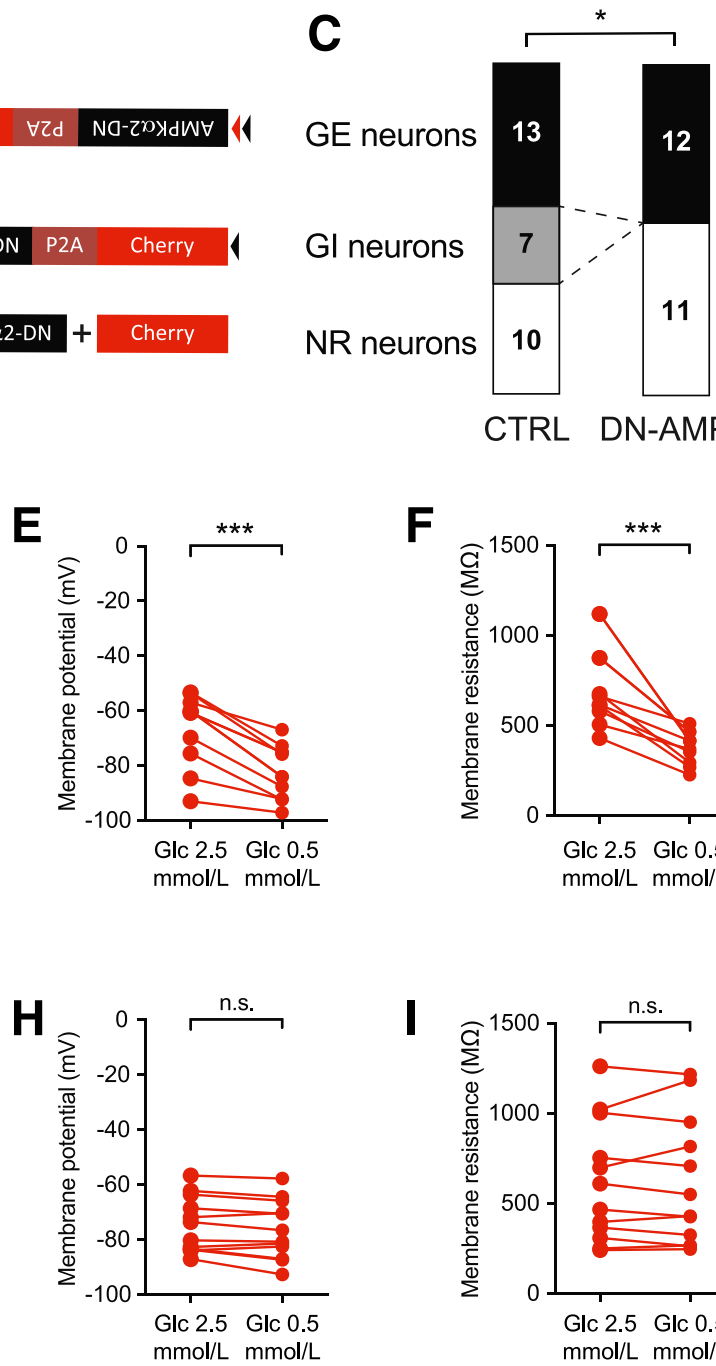

CTRL DN-AMPK
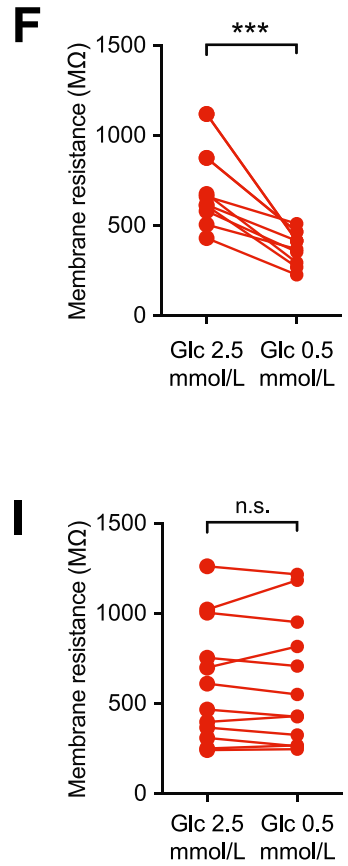

Figure 2-Loss of GI neurons after suppression of AMPK activity. A: rAAV8-DIO-AMPK-DN-mCherry was bilaterally injected in the VMN of Sf1-cre mice. B: Schematic representation of the rAAV8-DIO-AMPK-DN-mCherry viral construct. C: Comparison of the distribution of glucose responsive Sf1 neurons in control animals (CTRL from Fig. 1) and mice injected with the rAAV8-DIO-AMPK-DN-mCherry. GI neurons are no longer present when AMPK-DN is expressed (Fisher exact test; $P=0.0148$ for GI neurons proportion comparison). GE neurons $(D-F)$ $(n=12$ neurons in seven mice) and NR neurons $(G-l)(n=11$ neurons in six mice) subpopulations are still present and display similar features as GE and NR neurons from control animals (see Fig. 1). J: Experimental approach. rAAV8-DIO-EGFP was bilaterally injected in the VMN of Sf1-cre;AMPK $\alpha 1^{\text {lox/lox }} \alpha 2^{\text {lox/lox }}$ mice. K: Distribution of the glucose-responsive Sf1 neurons of the VMN $(n=12$ neurons in 8 mice and $n=$ 16 neurons in 10 mice for GE and NR, respectively). GI neurons were no longer detected in the mutant mice (Fisher exact test; $P=0.0107$ for GI neurons proportion compared with control conditions). Before-after graphs show individual values. Two-tailed paired $t$ test was used. ${ }^{*} P<$ $0.05 ;{ }^{\star \star} P<0.01$, and ${ }^{\star \star \star} P<0.001$. 

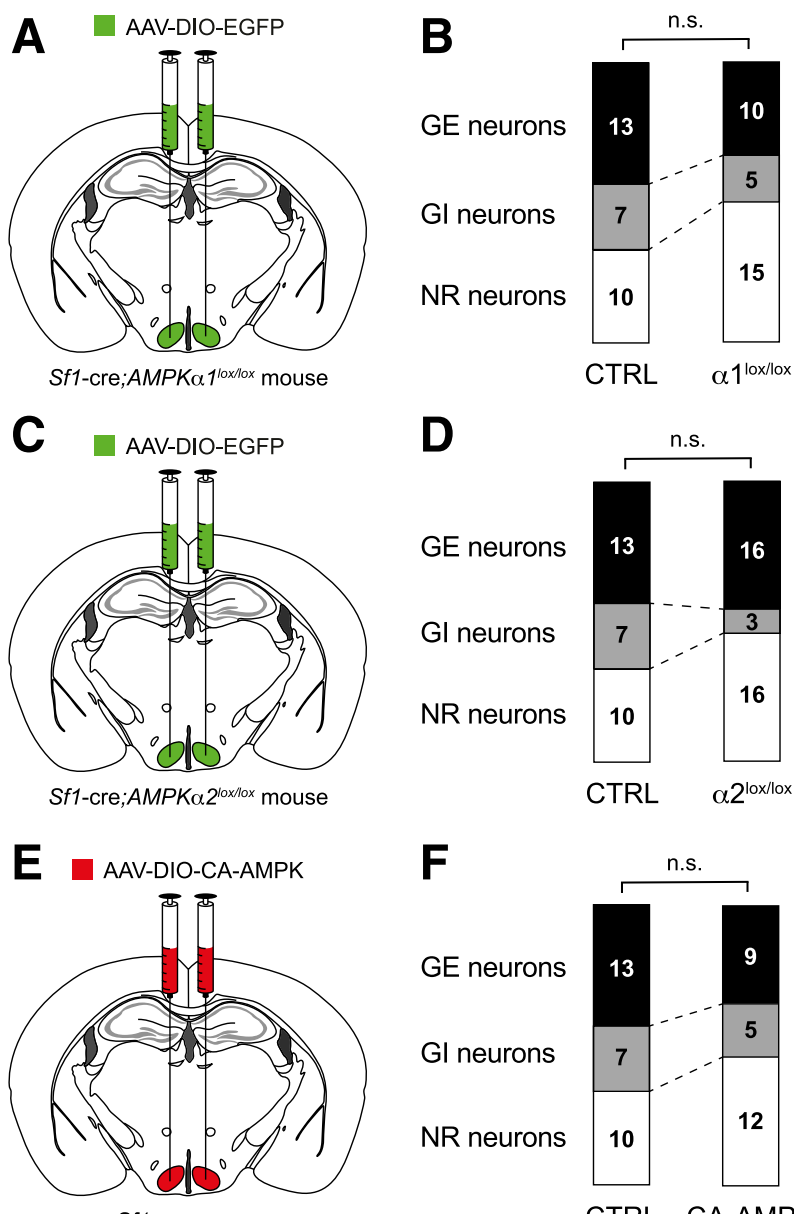

Sf1-cre mouse

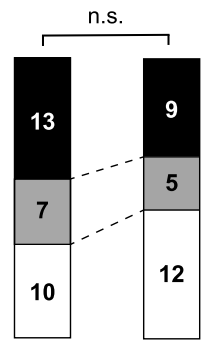

CTRL CA-AMPK

Figure 3-AMPK $\alpha$ subunits have redundant role in Gl neurons activation. A: rAAV8-DIO-EGFP was bilaterally injected in the VMN of Sf1-cre;AMPK $\alpha 1^{\text {lox/lox }}$ mice. $B$ : Distribution of the glucose-responsive Sf1 neurons in the absence of AMPK $\alpha 1$ subunit $(n=10 \mathrm{GE}, 5 \mathrm{GI}$, and $15 \mathrm{NR}$ neurons in 7,4 , and 10 mice, respectively). The distribution does not differ from that observed in control mice (Fisher exact test; $P>0.05$ ). C: rAAV8-DIO-EGFP was bilaterally injected in the VMN of Sf1-cre;AMPK $\alpha 2^{\text {lox/lox }}$ mice. $D$ : Distribution of the glucose-responsive $S f 1$ neurons in the absence of AMPK $\alpha 2$ subunit $(n=16 \mathrm{GE}, 3 \mathrm{GI}$, and $16 \mathrm{NR}$ neurons in eight, three, and nine mice, respectively). The distribution does not differ from that observed in control mice (Fisher exact test; $P>0.05)$. E: rAAV8-DIO-CA-AMPK-mCherry was bilaterally injected in the VMN of Sf1-cre mice. F: Distribution of the glucose-responsive Sf1 neurons in the presence of an AMPK-CA ( $n=9 \mathrm{GE}, 5 \mathrm{Gl}$, and $12 \mathrm{NR}$ neurons in six, four, and eight mice, respectively). The distribution does not differ from that observed in control mice (Fisher exact test; $P>0.05$ ). ${ }^{\star} P<$ $0.05,{ }^{\star \star} P<0.01$, and ${ }^{* \star *} P<0.001$.

resistance $(692.3 \pm 90.9 \mathrm{M} \Omega$ vs. $888.8 \pm 108.2 \mathrm{M} \Omega$ in $2.5 \mathrm{mmol} / \mathrm{L}$ and $0.5 \mathrm{mmol} / \mathrm{L}$ glucose, respectively; $P<0.01$ ), similar to those measured in control GI neurons. The electrophysiological properties of GE (Fig. $5 D-F$ ) and NR (Fig. 5J-L) neurons were not affected by Txn2 overexpression. The proportion of GE, GI, and NR neurons was also restored by Txn2 overexpression (Fig. 5C). Restoration of GI neuron activity was also obtained when using a lentiviral instead of an $A A V$ vector for $T x n 2$ reexpression (data not shown).

Thus, Txn2 expression is strongly reduced when AMPK activity is suppressed, and overexpression of Txn2 in Sf1 neurons is sufficient to restore the presence GI neurons. Overexpression of Txn2, however, does not modify the number of GE or NR neurons, suggesting that its role is specific for the response of GI neurons to hypoglycemia.

\section{AMPK Regulation of Txn2}

To investigate, in a cellular system, the link between AMPK and Txn2 expression, we used the GT1-7 neuronal cell line. These cells were transduced with recombinant lentiviruses encoding the dominant negative or the constitutively active form of AMPK and incubated in the presence of $0.1 \mathrm{mmol} / \mathrm{L}$ glucose. Real-time qPCR (Fig. $6 A$ ) and Western blot analysis (Fig. 6B-D) showed that overexpression of AMPK-DN reduced Txn 2 mRNA and protein expression. A reduction of acetyl-CoA-carboxylase (ACC) phosphorylation confirmed that AMPK-DN overexpression effectively reduced endogenous AMPK activity. In contrast, overexpression of the constitutively active form of AMPK did not impact Txn2 expression or ACC phosphorylation, indicating that the endogenous AMPK was maximally active in the presence of $0.1 \mathrm{mmol} / \mathrm{L}$ glucose.

Hypoglycemia induces reactive oxygen species (ROS) production, and Txn2 may be required to detoxify ROS to preserve hypoglycemia detection by GI neurons. To test this hypothesis, GT1-7 cells were first exposed to $0.1 \mathrm{mmol} / \mathrm{L}$ or $30 \mathrm{mmol} / \mathrm{L}$ glucose, and superoxide production was assessed by measuring MitoSOX red fluorescence intensity (Fig. 6E). We could confirm that exposure to low glucose levels induced ROS production (Fig. $6 F$ ). We then transduced GT1-7 cells with a recombinant lentivirus encoding a control or a Txn2-specific shRNA, which led to a very strong reduction of Txn2 mRNA (Fig. 6G). Exposing these cells to $0.1 \mathrm{mmol} / \mathrm{L}$ glucose induced a significantly higher increase in MitoSOX red staining when Txn2 expression was silenced (Fig. 6H).

Thus, AMPK controls the level of Txn2 expression; this was also observed when AMPK-DN was transduced in the Hepa1-6 mouse hepatoma cell line and therefore seems to be a general mechanism (Supplementary Fig. 3). In addition, suppressing Txn2 expression led to increased ROS production in the presence of low glucose concentrations. Whether exaggerated ROS production negatively impacts GI neurons activity is, however, to be further explored experimentally.

\section{Counterregulatory Response to Hypoglycemia in

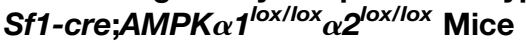

We analyzed glucose homeostasis and counterregulatory hormone secretion in control and Sf1-cre;AMPK $\alpha 1^{\text {lox/lox }}$ $\alpha 2^{l o x / l o x}$ mice. Glycemic levels were identical in both groups of mice in the fed and 24-h fasted states (Fig. $7 A$ ), and their fasted plasma glucagon levels were also identical (Fig. 7B). Their response to an insulin tolerance tests was also identical (Fig. 7C), indicating the same insulin sensitivity and counterregulatory response to 

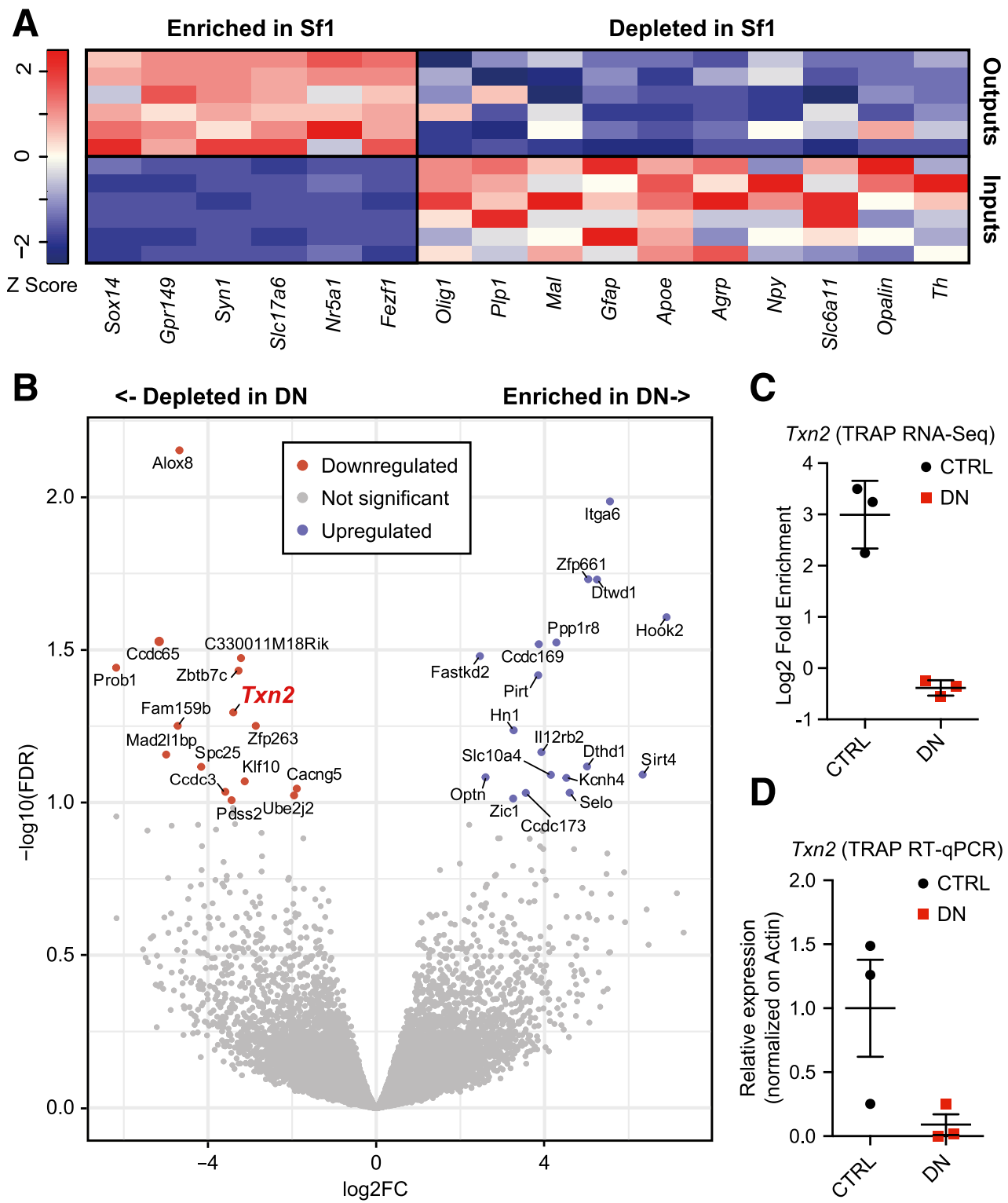

C

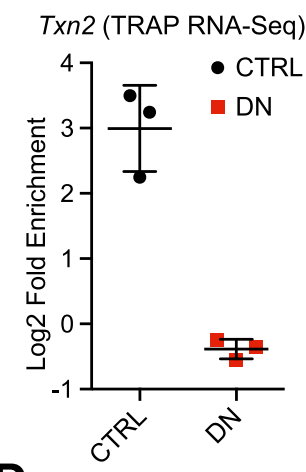

D

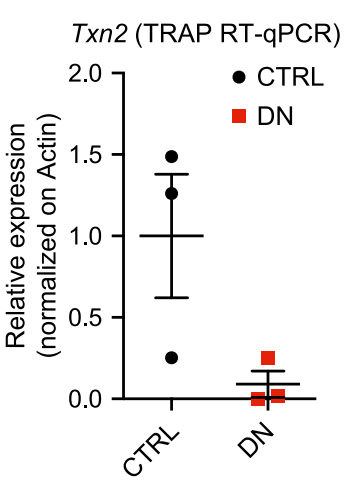

Figure 4-Txn2 expression is strongly suppressed in the absence of AMPK activity. Sf1-cre mice received intra-VMN administration of an AAV8-DIO-L10-EGFP (control) or of the same virus and an rAAV8-DIO-AMPK-DN-mCherry (DN). After transcribing ribosome affinity purification was performed on VMN lysates and RNA sequencing analysis, gene enrichment in the immunoprecipitated fraction over total lysate was calculated. $A$ : Heat map shows enrichment for VMN neuron mRNAs in the immunoprecipitated fraction (output) compared with the total lysate (input) and specific depletion in mRNAs expressed in non-VMN neurons, in glial cells or oligodendrocytes. $B$ : Volcano plot shows the mRNAs whose expression is significantly increased or downregulated in VMN Sf1 neurons expressing AMPK-DN. The $x$-axis shows logtwofold-changes in expression and the $y$-axis the log odds of a gene being differentially expressed (two-way ANOVA). C: Relative expression of Txn2 in control (CTRL) and AMPK-DN-expressing Sf1 neurons (DN); data from the TRAP analysis of (B). D: Real-time (RT) qPCR analysis of Txn2 expression levels in CTRL and AMPK-DN- expressing Sf1 neurons from a second TRAP experiment.

normalize hypoglycemia. We next assessed plasma glucagon, epinephrine, and norepinephrine levels in response to insulin-induced hypoglycemia. Figure $7 D$ shows that the blood glucose levels in control and mutant mice were the same $60 \mathrm{~min}$ after saline injection and that hypoglycemia induced by insulin injections reached the same levels. The basal plasma levels of glucagon, epinephrine, and norepinephrine were identical between control and mutant mice, as were their levels after induction of hypoglycemia (Fig.
$7 E-G$ ); insulin-induced glucagon secretion was also not different between female control and mutant mice (data not shown). To confirm these results, we measured plasma glucagon at the end of a hyperinsulinemichypoglycemic clamp. The same rates of glucose infusion were required to maintain hypoglycemia in control and Sf1-cre;AMPK $\alpha 1^{\text {lox/lox }} \alpha 2^{\text {lox/lox }}$ animals, and their plasma glucagon levels measured at the end of the clamp were similar (Fig. $7 H-J$ ). 


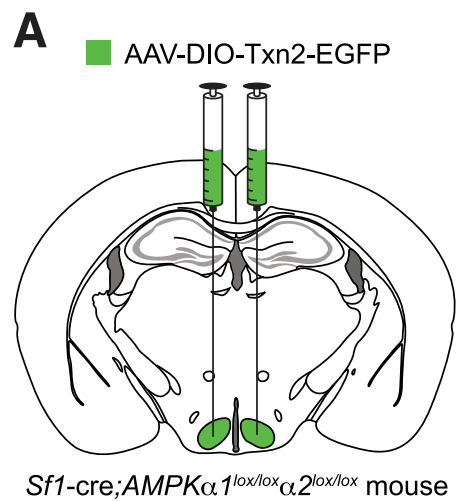

D

\section{GE neurons}

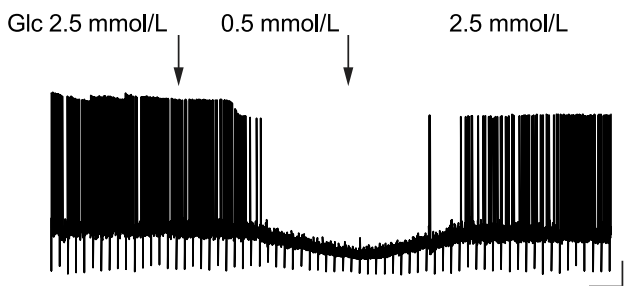

G

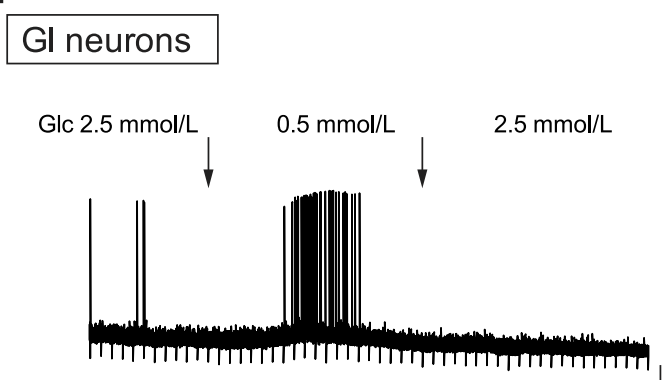

J

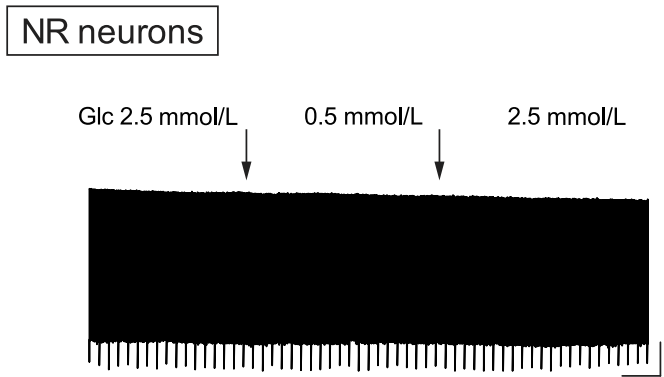

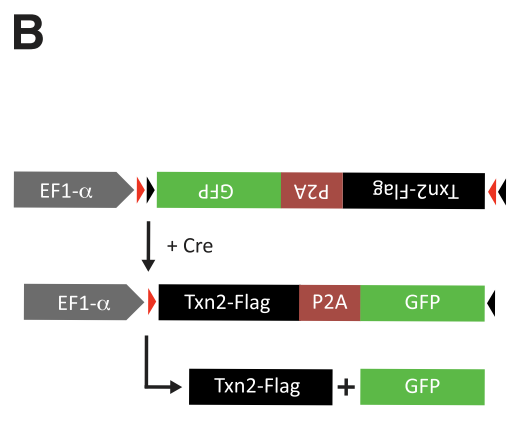

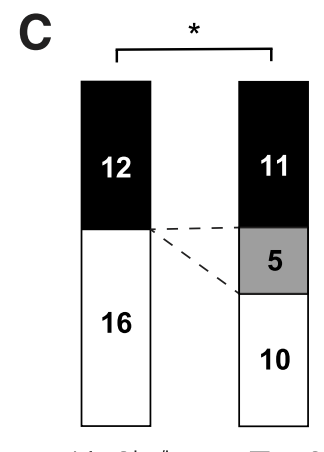

$\alpha 1 / \alpha 2^{\text {lox/lox }}+\operatorname{Txn} 2$
GE neurons

GI neurons

NR neurons
E

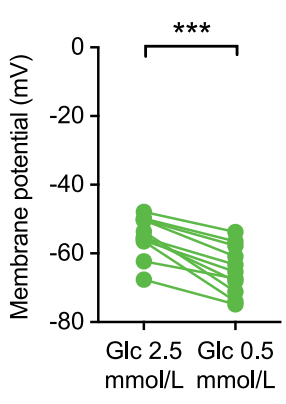

H

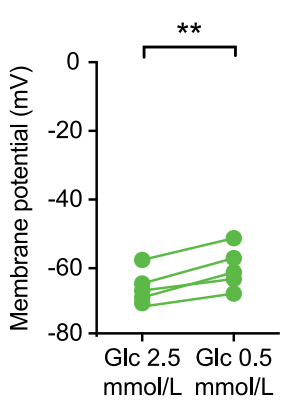

K

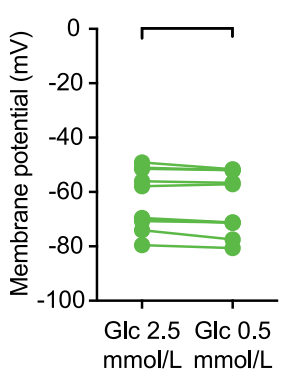

E

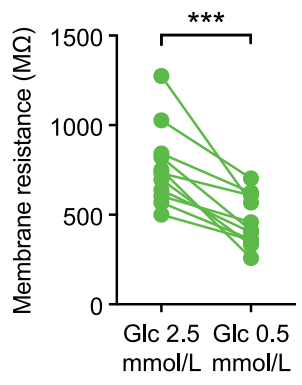

I

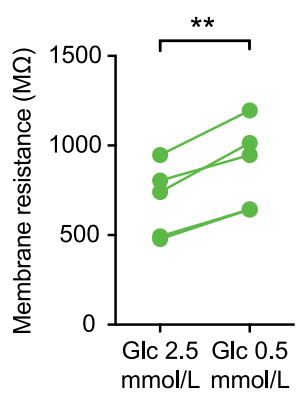

L

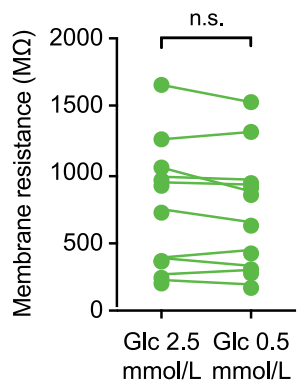

Figure 5-Expression of Txn2 in Sf1-cre;AMPK $\alpha 1^{\text {lox/lox }} \alpha 2^{\text {lox/lox }}$ restores GI neuron activity. A: rAAV8-DIO-Txn2-EGFP was bilaterally injected in the VMN of Sf1-cre;AMPK $\alpha 1^{\text {lox/lox }} \alpha 2^{\text {lox/lox }}$ mice. B: Schematic representation of the rAAV8-DIO-Txn2-EGFP viral construct. C: Distribution of glucose responsive Sf1 neurons from Sf1-cre;AMPK $\alpha 1^{\text {lox/lox }} \alpha 2^{\text {lox/lox }}$ mice (from Fig. $2 K$ ) and from the same mice having been injected with the Txn2 virus ( $n=11 \mathrm{GE}, 5 \mathrm{Gl}$ and $10 \mathrm{NR}$ neurons in seven, four, and seven mice, respectively). Fisher exact test; $P>0.05$ for $\mathrm{Gl}$ neurons proportion comparison. Txn2-expressing GE neurons $(D-F), \mathrm{Gl}(\mathrm{G}-l)$, and NR $(J-L)$ neurons exhibit similar electrophysiological characteristics as GE, GI, and NR subpopulations monitored in control animals (see Fig. 1). Before-after graphs show individual values. Two-tailed paired $t$ test. ${ }^{*} P<0.05,{ }^{* \star} P<0.01$. and ${ }^{* \star \star} P<0.001$. 




C

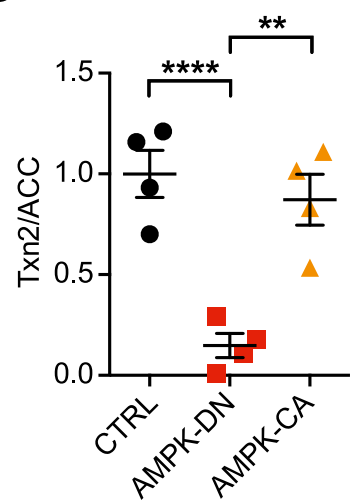

$\mathbf{F}$

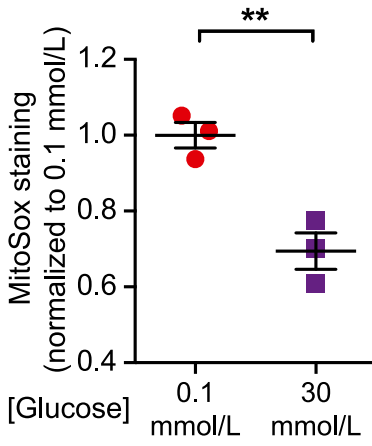

B

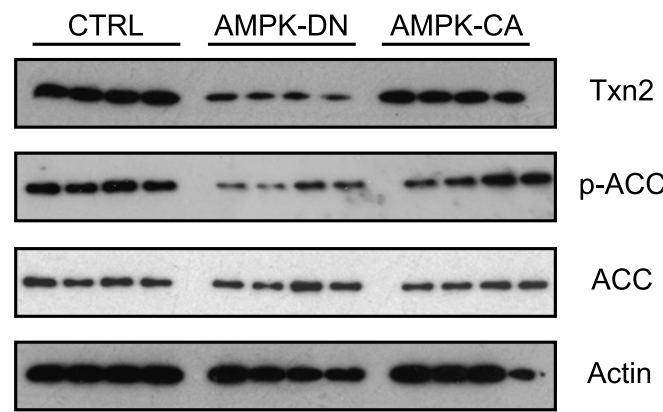

D

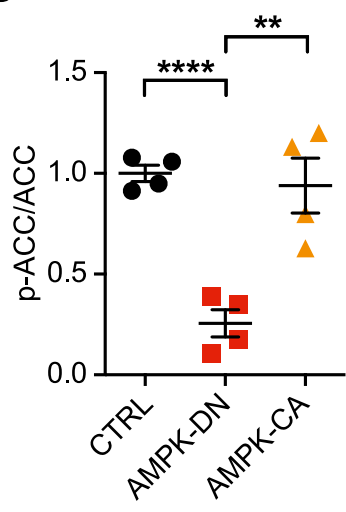

G

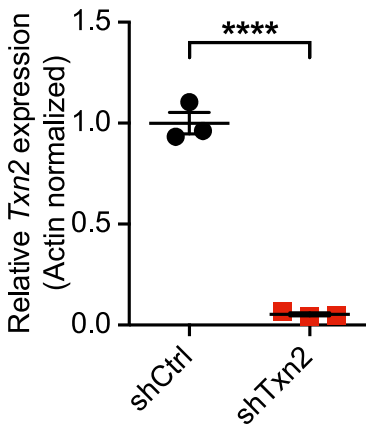

E

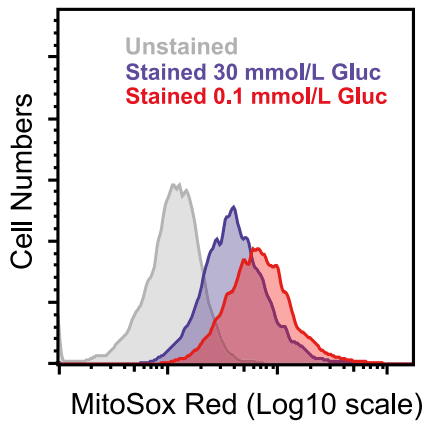

H

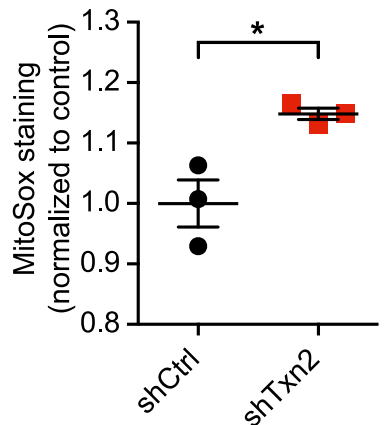

Figure 6-Txn2 expression is controlled by AMPK and reduces ROS accumulation. GT1-7 cells were transduced with a control lentivector or a lentivector encoding AMPK-DN or AMPK-CA. The cells were incubated in $0.1 \mathrm{mmol} / \mathrm{L}$ glucose for $48 \mathrm{~h}$ before RNA and protein extraction. $A$ : Txn2 expression in GT1-7 cells transfected with a control vector or a vector encoding $A M P K-D N$ or $A M P K-C A(t$ test, $P<0.02)$. B: Western blot analysis of Txn2, phosphorylated (p)-ACC, total ACC, and actin from cells transduced with the mentioned vectors. $C$ and $D: Q u a n t i t a t i o n$ of the Western blots of $(B)$. E: MitoSOX red staining of GT1-7 cells incubated in the presence of $0.1 \mathrm{mmol} / \mathrm{L}$ or $30 \mathrm{mmol} / \mathrm{L}$ glucose for $48 \mathrm{~h}$. Unstained cells were used as control. Incubation in low glucose increased superoxide production. F: Quantitation of the MitoSOX red staining of figure (E). G: Txn2 mRNA levels in GT1-7 cells transduced with a control or a Txn2-specific shRNA. H: MitoSOX red staining of GT1-7 cells transduced with a control or Txn2-specific shRNA and incubated in $0.1 \mathrm{mmol} / \mathrm{L}$ glucose for $48 \mathrm{~h}$. For all panels, $t$ test, ${ }^{\star} P<0.05$, ${ }^{\star \star} P<0.01$, and ${ }^{* \star *} P<0.001$.

The VMN and AMPK expressed in Sf1 neurons have also been involved in the control of feeding (38). We thus tested feeding of CTRL and Sf1-cre;AMPK $\alpha 1^{\text {lox } / l o x}$ $\alpha 2^{\text {lox/lox }}$ mice over a 48 -h period (Fig. $7 K$ ), and no difference in the cumulative food absorption over time could be observed. Similarly, the rate of refeeding after an 18-h fast was not different between both groups of mice (Fig. $7 L$ ).

\section{DISCUSSION}

In the current study, we used a combination of genetic and electrophysiological approaches to dissect the role of AMPK $\alpha 1$ and $\alpha 2$ subunits in glucose sensing by VMN Sf1 neurons. We found that suppressing AMPK activity led to the selective depletion of GI neurons, without affecting the presence of GE neurons. These observations allowed us to further investigate two aspects of hypoglycemia sensing. 

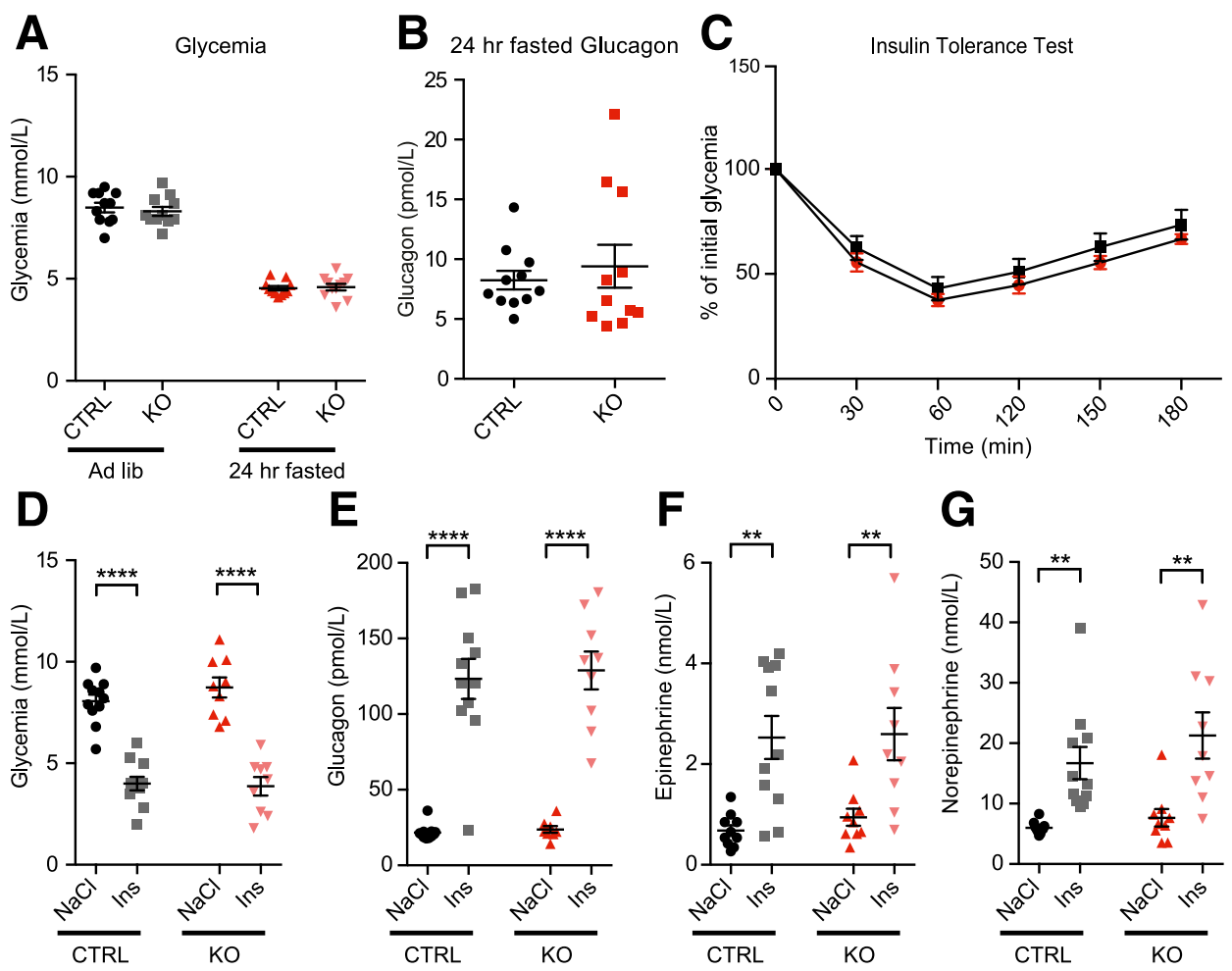

$\mathrm{E}$

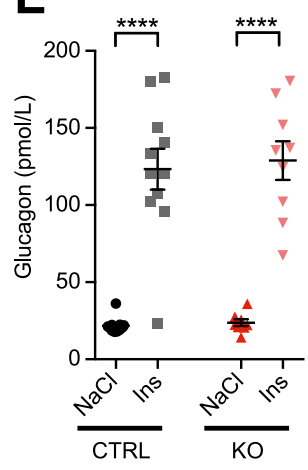

$\mathbf{F}$

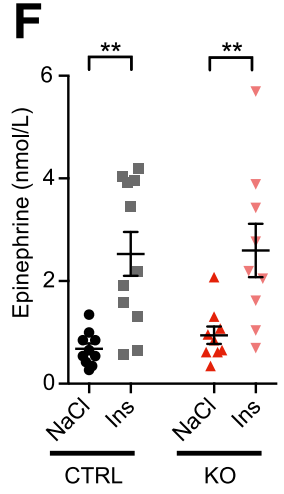

G
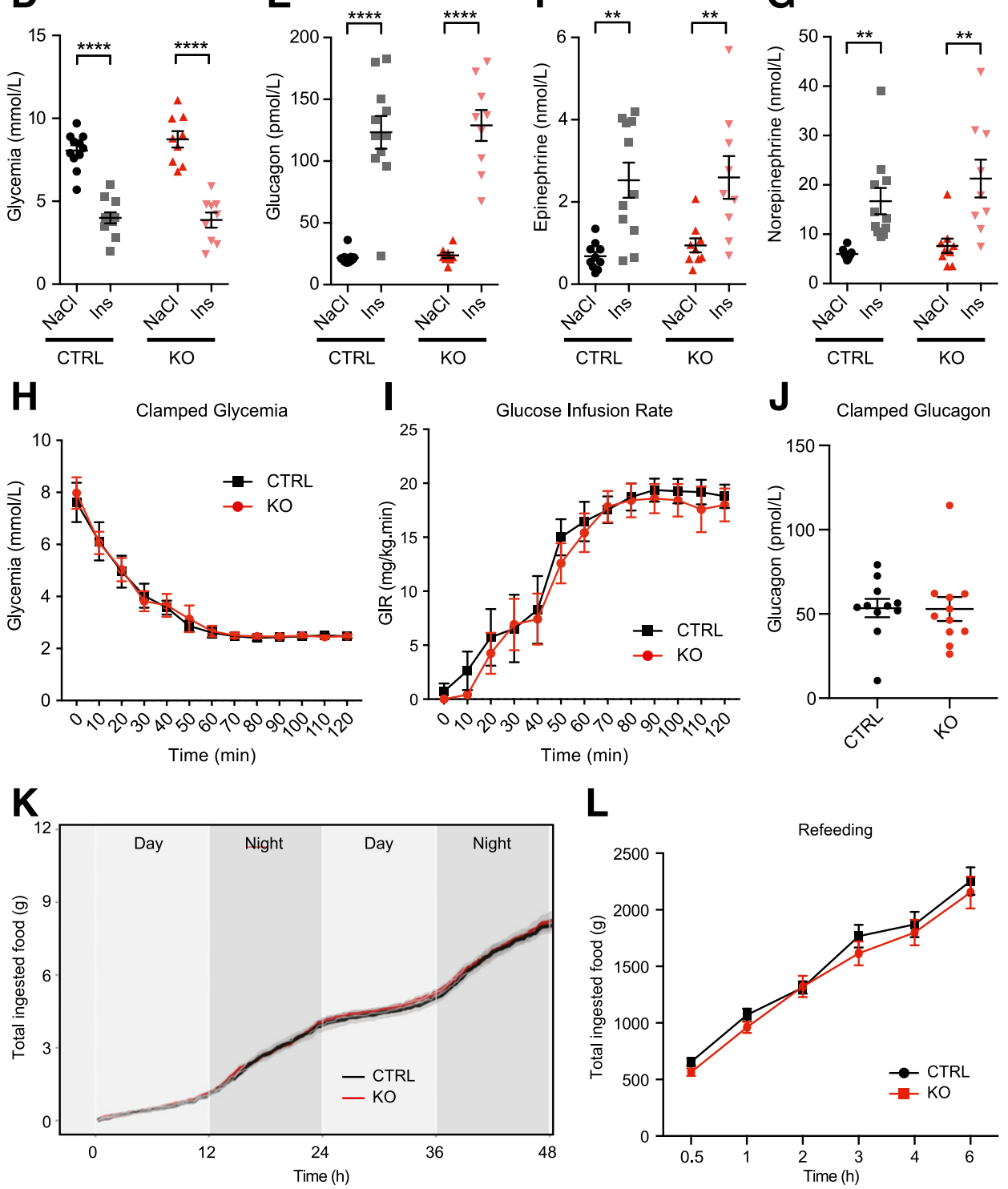

Figure 7-Normal counterregulation and feeding in Sf1-cre;AMPK $\alpha 1^{\text {lox/lox }} \alpha 2^{\text {lox/lox }}$ mice. A: Random fed and 24-h fasted glycemia in Sf1cre;AMPK $\alpha 1^{\text {lox/lox }} \alpha 2^{\text {lox/lox }}$ knock-out (KO) and control (CTRL) mice $(n=22,11 \mathrm{CTRL}, 11 \mathrm{KO})$. B: Plasma glucagon levels in CTRL and KO mice after 24-h fast. C: Insulin tolerance test in CTRL and KO mice $(n=20,10 \mathrm{CTRL}, 10 \mathrm{KO})$. Glycemia $(D)$, plasma glucagon $(E)$, epinephrine $(F)$, and norepinephrine $(G) 60$ min after i.p. saline or insulin $(0.8 \mathrm{units} / \mathrm{kg})$ injections $(n=20,11 \mathrm{CTRL}, 9 \mathrm{KO})$. H: Hyperinsulinemic-hypoglycemic clamps were performed to maintain glycemic levels at $\sim 2.5 \mathrm{mmol} / \mathrm{L}$ glucose. The glucose infusion rates were identical to maintain hypoglycemia ( () , and the plasma glucagon levels were the same at the end of the clamp in CTRL and KO mice $(J)(n=23,11 \mathrm{CTRL}, 12 \mathrm{KO}) . K$ : Identical food intake between CTRL and KO mice in ad libitum condition monitored for $48 \mathrm{~h}(n=10,5 \mathrm{CTRL}, 5 \mathrm{KO}$; two-way ANOVA, $P=$ $0.7521)$. $L$ : Refeeding experiment after overnight fast shows no difference between CTRL and KO mice in food intake $(n=18,7 \mathrm{CTRL}, 11 \mathrm{KO})$ (two-way ANOVA, $P=0.4760$ ). For all panels, two-tailed $t$ test was used. ${ }^{\star} P<0.05$, ${ }^{\star \star} P<0.01$, and ${ }^{\star \star \star} P<0.001$. 
First, we investigated the molecular components participating in hypoglycemia-induced neuron activation, and second, the impact of suppressing GI neurons activity in physiological regulations. We found that Txn2 expression was strongly decreased when AMPK was inactivated, and we showed that reexpression of Txn2 in Sf1 neurons of Sf1$c r e ; A M P K \alpha 1^{\text {lox } / l o x} \alpha 2^{\text {lox/lox }}$ mice restored GI neuron activity. We further showed that silencing Txn2 led to exaggerated ROS production in cells exposed to low glucose concentrations. This suggests that the role of AMPK is to maintain sufficient levels of Txn2 expression to prevent the deleterious effect of ROS on GI neuron function. Importantly, in physiological studies, we found that suppression of VMN GI neuron activity had no impact on the counterregulatory hormone response or on feeding.

AMPK is an evolutionary conserved, ubiquitously expressed energy-sensing kinase that is activated in conditions such as fasting, hypoglycemia, or hypoxia $(39,40)$. In the central nervous system, in particular in the VMN plus arcuate nucleus, it has been reported that its activation by hypoglycemia or 2DG-induced neuroglucopenia is involved in the control of feeding (41) and the secretion of counterregulatory hormones (15). The VMN, however, consists of several neuronal subpopulations, characterized by the expression of different transcription factors, neurotransmitter receptors, or neuropeptides $(42,43)$ and by their differential glucose responsiveness $(30,44)$. Thus, expression of AMPK in different subpopulations may have different physiological impacts that are not revealed in the studies mentioned above. Here, we investigated the role of the AMPK $\alpha 1$ and $\alpha 2$ subunits specifically in glucose sensing in Sf1 neurons of the VMN. Overexpression of a dominant negative form of the kinase or genetic inactivation of both AMPK $\alpha 1$ and $\alpha 2$ genes suppressed GI activity. These observations are in agreement with published data showing that AMPK is required for hypoglycemia detection by GI neurons of the VMH (17). They, however, extend these previous data by showing that both $\alpha 1$ and $\alpha 2$ isoforms similarly contribute to GI neurons glucose sensing. They also show that the loss of AMPK activity does not impact GE neuron response. Thus, AMPK is selectively required for the activity of GI neurons.

AMPK regulates multiple cellular functions by direct protein phosphorylation (45) or transcriptional regulation (46). Here, we identified Txn2 as an AMPK-regulated mRNA. Txn2 is part of a redox system that detoxifies ROS and reactive nitrogen species (32); it is distinct from Txn1, a cytosolic enzyme (47). Hypoglycemia is known to induce ROS production in the brain and in the VMN $(37,48,49)$, whereas hyperglycemia reduces ROS production in a UCP2-dependent manner in GE neurons of the VMN (50). ROS levels have also been shown to have antagonistic effects on NPY/Agrp and POMC neurons, reducing the activity of the first ones and stimulating the activity of the second ones (51). Thus, depending on the cellular context and also on their intracellular concentrations, ROS can have positive signaling effects on neuron activity and gene expression or toxic effects leading to cell apoptosis (52). The concept of mitohormesis describes that ROS can shift from positive regulators of cellular function to inducers of cell death (53).

In GT1-7 cells shifted from 30 to $0.1 \mathrm{mmol} / \mathrm{L}$ glucose, there is a strong induction of superoxide production, in agreement with the fact that hypoglycemia increases ROS production (49) by shifting metabolism to $\beta$-oxidation (48). If increased ROS production is associated with a normal firing activity of neurons, the loss of GI response when Txn2 is suppressed, and restoration of such activity by its reexpression, suggests that $\mathrm{Txn} 2$ plays a protective role against a toxic effect of ROS. How ROS production can prevent activation of GI neurons is not known. However, one possibility is that superoxide ions produced in the mitochondria can be transported into the cytosol, where they can react with $\mathrm{NO}$ to produce peroxynitrite $\left(\mathrm{ONOO}^{-}\right)$ (52). Because NO production is required for GI neuron activity (18), this reaction may dampen the GI neuron response.

The fact that Txn2 restores GI neuron activity in the absence of AMPK activity suggests that the role of this kinase is not in the acute signaling of hypoglycemia. Instead, its role may be to protect against the deleterious effects of ROS on signal transduction by controlling the level of expression of antioxidant proteins, in particular, Txn2. In support of this hypothesis is the observation that among the other genes that were downregulated when AMPK-DN was overexpressed was Pdss2 (or Coq1B), which codes for an enzyme involved in the biosynthesis of the prenyl side chain of coenzyme $Q$. This cofactor transports electrons from complex I to complex II of the electron transport chain and, in its reduced form, also acts as an antioxidant (54). Decreased expression of Pdss2 and coenzyme $Q$ deficiency have been linked to increased ROS production and cell death $(55,56)$. Thus, AMPK activity may protect cells against ROS toxicity also by increasing the expression of this biosynthetic enzyme. Along the same line, a previous study performed in a rat model of hypoglycemia-associated autonomic failure showed that the loss of VMN GI neurons could be prevented by overexpressing the cytosolic form of thioredoxin, Txn1 (57).

One of the initial goals of our study was to identify the physiological functions controlled by VMN GI neurons, in particular whether selective impairment in the counterregulatory response to hypoglycemia could be demonstrated. However, plasma glucagon in fasted mice and insulin tolerance tests were similar in control and Sf1$c r e ; A M P K \alpha 1^{l o x / l o x} \alpha 2^{l o x / l o x}$ mice. Similarly, glucagon, epinephrine, and norepinephrine secretion in response to insulin-induced hypoglycemia, as well as plasma glucagon levels at the end of a hyperinsulinemic-hypoglycemic clamp, were identical. Thus, suppression of GI activity in the VMN does not reduce hypoglycemia-induced counterregulatory hormone secretion. Another aspect of the response to hypoglycemia is the stimulation of food intake, but we could not observe any difference in food intake 
measured over a 48 -h period or in the rate of refeeding after an overnight fast.

Together, these results indicate that the intrinsic glucose sensitivity of GI neurons of the VMN is dispensable for the physiological response to hypoglycemia. These neurons are part of a circuit that includes afferent, glucose-sensing neurons directly sensitive to small variations in blood glucose concentrations, such as those present in the hepatoportal vein area and of the nucleus of the tractus solitarius. In this circuit, these peripheral sensing cells probably have a primary role in triggering the counterregulatory response to hypoglycemia. The glucose-sensing capacity the VMN GI neurons may, thus, represent a failsafe system, activated only in case of failure of the peripheral sensing cells leading to development of brain hypoglycemia.

In summary, the current study establishes that AMPK $\alpha 1$ and $\alpha 2$ subunits play redundant roles in allowing the hypoglycemia detection capacity of Sf1 GI neurons. It identifies Txn2 as a necessary element in the GI neurons response to hypoglycemia, which can restore GI activity even in the absence of AMPK. This suggests that the primary role of AMPK in GI neurons is to control the expression of $T x n 2$, and possibly other enzymes, participating in the antioxidant protection against ROS produced during hypoglycemia. Finally, these data show that the intrinsic hypoglycemia-sensing capacity of VMN Sf1 neurons is dispensable for triggering counterregulatory hormone secretion or feeding and rather represents a fail-safe system in case of failure of peripheral hypoglycemia sensing system allowing development of hypoglycemia in the VMN.

Acknowledgments. The authors thank Dr. Sylvain Pradervand and the members of the Genome Technology Facility of the University of Lausanne for their help with RNA sequencing and data analysis and Dr. Frédéric Preitner and Anabela da Costa of the Scientic Service of the Center for Integrative Genomics (SSC), University of Lausanne, for performing the hypoglycemic clamps. The authors thank Drs. Brad Lowell and Barbara Kahn (Harvard University) and Dr. Dong Kong (Tufts University) for the gift of the AMPK-DN and AMPK-CA expression plasmids and recombinant AAVs.

Funding. The present work was supported by a European Research Council Advanced Grant (INTEGRATE, No. 694798) and a Swiss National Science Foundation grant (310030-182496) to B.T., and has received funding from the Innovative Medicines Initiative 2 Joint Undertaking under grant agreement № 777460 (HypoRESOLVE). The Joint Undertaking receives support from the European Union's Horizon 2020 Research and Innovation Programme and EFPIA and T1D Exchange, JDRF, International Diabetes Federation, and The Leona M. and Harry B. Helmsley Charitable Trust.

Duality of Interest. No potential conflicts of interest relevant to this article were reported.

Author Contributions. S.Q., G.L., D.B., and S.M. performed the experiments. S.Q., G.L., and B.T. designed the experiments. S.Q., G.L., and B.T. analyzed the data and wrote the manuscript. B.V. and M.F. provided genetically modified mice and reviewed the manuscript. B.T. conceived the project. B.T. takes responsibility for the content of the manuscript. B.T. is the guarantor of this work and, as such, had full access to all the data in the study and takes responsibility for the integrity of the data and the accuracy of the data analysis.

\section{References}

1. Verberne AJ, Sabetghadam A, Korim WS. Neural pathways that control the glucose counterregulatory response. Front Neurosci 2014;8:38

2. Cryer PE. Mechanisms of hypoglycemia-associated autonomic failure in diabetes. N Engl J Med 2013;369:362-372

3. Marty N, Dallaporta M, Thorens B. Brain glucose sensing, counterregulation, and energy homeostasis. Physiology (Bethesda) 2007;22:241-251

4. Labouèbe G, Boutrel B, Tarussio D, Thorens B. Glucose-responsive neurons of the paraventricular thalamus control sucrose-seeking behavior. Nat Neurosci 2016;19:999-1002

5. Lamy CM, Sanno H, Labouèbe G, et al. Hypoglycemia-activated GLUT2 neurons of the nucleus tractus solitarius stimulate vagal activity and glucagon secretion. Cell Metab 2014;19:527-538

6. Routh VH. Glucose-sensing neurons: are they physiologically relevant? Physiol Behav 2002;76:403-413

7. Borg MA, Sherwin RS, Borg WP, Tamborlane WV, Shulman Gl. Local ventromedial hypothalamus glucose perfusion blocks counterregulation during systemic hypoglycemia in awake rats. J Clin Invest 1997;99:361-365

8. Borg WP, Sherwin RS, During MJ, Borg MA, Shulman Gl. Local ventromedial hypothalamus glucopenia triggers counterregulatory hormone release. Diabetes 1995;44:180-184

9. Tong $Q$, Ye C, McCrimmon RJ, et al. Synaptic glutamate release by ventromedial hypothalamic neurons is part of the neurocircuitry that prevents hypoglycemia. Cell Metab 2007;5:383-393

10. Meek TH, Nelson JT, Matsen ME, et al. Functional identification of a neurocircuit regulating blood glucose. Proc Natl Acad Sci U S A 2016;113:E2073E2082

11. Bentsen MA, Mirzadeh Z, Schwartz MW. Revisiting how the brain senses glucose-and why. Cell Metab 2019;29:11-17

12. Dallaporta M, Himmi T, Perrin J, Orsini JC. Solitary tract nucleus sensitivity to moderate changes in glucose level. Neuroreport 1999;10:2657-2660

13. Garfield AS, Shah BP, Madara JC, et al. A parabrachial-hypothalamic cholecystokinin neurocircuit controls counterregulatory responses to hypoglycemia. Cell Metab 2014;20:1030-1037

14. Lindberg $D$, Chen P, Li C. Conditional viral tracing reveals that steroidogenic factor 1-positive neurons of the dorsomedial subdivision of the ventromedial hypothalamus project to autonomic centers of the hypothalamus and hindbrain. J Comp Neurol 2013;521:3167-3190

15. McCrimmon RJ, Shaw M, Fan X, et al. Key role for AMP-activated protein kinase in the ventromedial hypothalamus in regulating counterregulatory hormone responses to acute hypoglycemia. Diabetes 2008;57:444-450

16. Fioramonti X, Marsollier N, Song Z, et al. Ventromedial hypothalamic nitric oxide production is necessary for hypoglycemia detection and counterregulation. Diabetes 2010;59:519-528

17. Hirschberg PR, Sarkar P, Teegala SB, Routh VH. Ventromedial hypothalamus glucose-inhibited neurones: a role in glucose and energy homeostasis? J Neuroendocrinol 2020;32:e12773

18. Fioramonti X, Song Z, Vazirani RP, Beuve A, Routh VH. Hypothalamic nitric oxide in hypoglycemia detection and counterregulation: a two-edged sword. Antioxid Redox Signal 2011;14:505-517

19. Boudaba N, Marion A, Huet C, Pierre R, Viollet B, Foretz M. AMPK re-activation suppresses hepatic steatosis but its downregulation does not promote fatty liver development. EBioMedicine 2018;28:194-209

20. Kong D, Dagon Y, Campbell JN, et al. A postsynaptic AMPK $\rightarrow$ p21-activated kinase pathway drives fasting-induced synaptic plasticity in AgRP neurons. Neuron 2016;91:25-33

21. Nectow AR, Schneeberger $M$, Zhang $H$, et al. Identification of a brainstem circuit controlling feeding. Cell 2017;170:429-442.e11

22. Salmon P, Trono D. Production and titration of lentiviral vectors. Curr Protoc Neurosci 2006;Chapter 4:Unit 4.21 
23. Heiman $M$, Schaefer A, Gong S, et al. A translational profiling approach for the molecular characterization of CNS cell types. Cell 2008;135:738-748

24. Anders S, Pyl PT, Huber W. HTSeq-a Python framework to work with highthroughput sequencing data. Bioinformatics 2015;31:166-169

25. Modi H, Cornu M, Thorens B. Glutamine stimulates biosynthesis and secretion of insulin-like growth factor 2 (IGF2), an autocrine regulator of beta cell mass and function. J Biol Chem 2014;289:31972-31982

26. Tarussio $D$, Metref $S$, Seyer $P$, et al. Nervous glucose sensing regulates postnatal $\beta$ cell proliferation and glucose homeostasis. J Clin Invest 2014;124:413-424

27. Dunand M, Gubian D, Stauffer M, Abid K, Grouzmann E. High-throughput and sensitive quantitation of plasma catecholamines by ultraperformance liquid chromatography-tandem mass spectrometry using a solid phase microwell extraction plate. Anal Chem 2013;85:3539-3544

28. Burcelin R, Thorens B. Evidence that extrapancreatic GLUT2-dependent glucose sensors control glucagon secretion. Diabetes 2001;50:1282-1289

29. Silver IA, Erecińska M. Extracellular glucose concentration in mammalian brain: continuous monitoring of changes during increased neuronal activity and upon limitation in oxygen supply in normo-, hypo-, and hyperglycemic animals. J Neurosci 1994;14:5068-5076

30. Song Z, Levin BE, McArdle JJ, Bakhos N, Routh VH. Convergence of pre- and postsynaptic influences on glucosensing neurons in the ventromedial hypothalamic nucleus. Diabetes 2001;50:2673-2681

31. Kurrasch DM, Cheung CC, Lee FY, Tran PV, Hata K, Ingraham HA. The neonatal ventromedial hypothalamus transcriptome reveals novel markers with spatially distinct patterning. J Neurosci 2007;27:13624-13634

32. Netto LES, Antunes F. The roles of peroxiredoxin and thioredoxin in hydrogen peroxide sensing and in signal transduction. Mol Cells 2016;39:65-71

33. Balsera M, Buchanan BB. Evolution of the thioredoxin system as a step enabling adaptation to oxidative stress. Free Radic Biol Med 2019;140:28-35 34. Hu C, Zhang H, Qiao Z, Wang Y, Zhang P, Yang D. Loss of thioredoxin 2 alters mitochondrial respiratory function and induces cardiomyocyte hypertrophy. Exp Cell Res 2018;372:61-72

35. Matsuzawa A. Thioredoxin and redox signaling: roles of the thioredoxin system in control of cell fate. Arch Biochem Biophys 2017;617:101-105

36. Patocková J, Marhol P, Tůmová E, et al. Oxidative stress in the brain tissue of laboratory mice with acute post insulin hypoglycemia. Physiol Res 2003;52:131-135

37. Pauliina Markkula S, Lyons D, Yueh CY, et al. Intracerebroventricular catalase reduces hepatic insulin sensitivity and increases responses to hypoglycemia in rats. Endocrinology 2016;157:4669-4676

38. Viskaitis P, Irvine EE, Smith MA, et al. Modulation of SF1 neuron activity coordinately regulates both feeding behavior and associated emotional states. Cell Rep 2017;21:3559-3572

39. Carling D. The AMP-activated protein kinase cascade-a unifying system for energy control. Trends Biochem Sci 2004;29:18-24

40. Viollet B, Horman S, Leclerc J, et al. AMPK inhibition in health and disease. Crit Rev Biochem Mol Biol 2010;45:276-295
41. Minokoshi Y, Alquier T, Furukawa N, et al. AMP-kinase regulates food intake by responding to hormonal and nutrient signals in the hypothalamus. Nature 2004; 428:569-574

42. Krause WC, Ingraham HA. Origins and functions of the ventrolateral VMH: a complex neuronal cluster orchestrating sex differences in metabolism and behavior. Adv Exp Med Biol 2017;1043:199-213

43. McClellan KM, Parker KL, Tobet S. Development of the ventromedial nucleus of the hypothalamus. Front Neuroendocrinol 2006;27:193-209

44. Kang L, Routh VH, Kuzhikandathil EV, Gaspers LD, Levin BE. Physiological and molecular characteristics of rat hypothalamic ventromedial nucleus glucosensing neurons. Diabetes 2004;53:549-559

45. Hardie DG. Minireview: the AMP-activated protein kinase cascade: the key sensor of cellular energy status. Endocrinology 2003;144:5179-5183

46. Collodet C, Foretz M, Deak M, et al. AMPK promotes induction of the tumor suppressor FLCN through activation of TFEB independently of mTOR. FASEB J 2019;33:12374-12391

47. Lu J, Holmgren A. The thioredoxin antioxidant system. Free Radic Biol Med 2014;66:75-87

48. Kajihara N, Kukidome D, Sada K, et al. Low glucose induces mitochondrial reactive oxygen species via fatty acid oxidation in bovine aortic endothelial cells. J Diabetes Investig 2017;8:750-761

49. Páramo B, Hernández-Fonseca K, Estrada-Sánchez AM, Jiménez N, HernándezCruz A, Massieu L. Pathways involved in the generation of reactive oxygen and nitrogen species during glucose deprivation and its role on the death of cultured hippocampal neurons. Neuroscience 2010;167:1057-1069

50. Toda C, Kim JD, Impellizzeri D, Cuzzocrea S, Liu ZW, Diano S. UCP2 regulates mitochondrial fission and ventromedial nucleus control of glucose responsiveness. Cell 2016;164:872-883

51. Diano S, Liu ZW, Jeong JK, et al. Peroxisome proliferation-associated control of reactive oxygen species sets melanocortin tone and feeding in diet-induced obesity. Nat Med 2011;17:1121-1127

52. Shadel GS, Horvath TL. Mitochondrial ROS signaling in organismal homeostasis. Cell 2015;163:560-569

53. Ristow M. Unraveling the truth about antioxidants: mitohormesis explains ROS-induced health benefits. Nat Med 2014;20:709-711

54. Wang Y, Hekimi S. The complexity of making ubiquinone. Trends Endocrinol Metab 2019;30:929-943

55. Quinzii CM, Garone C, Emmanuele V, et al. Tissue-specific oxidative stress and loss of mitochondria in CoQ-deficient Pdss2 mutant mice. FASEB J 2013;27: 612-621

56. Quinzii CM, López LC, Gilkerson RW, et al. Reactive oxygen species, oxidative stress, and cell death correlate with level of CoQ10 deficiency. FASEB J 2010;24: 3733-3743

57. Zhou C, Routh VH. Thioredoxin-1 overexpression in the ventromedial nucleus of the hypothalamus preserves the counterregulatory response to hypoglycemia during type 1 diabetes in male rats. Diabetes 2018;67:120-130 